\title{
Derivation, characterization and directed differentiation of a new human embryonic stem cell line from a Chinese blastocyst hatched by a non-contact laser system
}

\author{
Rongrong $\mathrm{Wu}^{1}$, Chenming $\mathrm{Xu}^{2}$, Liangbiao Chen ${ }^{3}$, Xiaoli Zhao ${ }^{1}$, Xing Yao ${ }^{4}$, Licheng $\mathrm{Dai}^{4}$, Fan Jin ${ }^{2}$, Ming Zhang ${ }^{1}$ \\ ${ }^{I}$ College of Life Science, Zhejiang University, Hangzhou 310058, China; ${ }^{2}$ In Vitro Fertilization Center of Women's Hospital, \\ College of Medicine, Zhejiang University, Hangzhou 310058, China; ${ }^{3}$ Institute of Genetics and Developmental Biology, Chinese \\ Academy of Science, Beijing 100101, China: ${ }^{4}$ Central Hospital of Huzhou, Huzhou 313000, China
}

Human embryonic stem cells (hESCs) possess enormous capability serving as an excellent model for human embryonic development, as a unique platform for pharmaceutical screening and as an unlimited resource for cell replacement therapy. Currently, worldwide attention has focused on the derivation of hESCs for clinical application. However, the majority of already established hESCs including NIH-approved lines have been directly or indirectly exposed to non-human materials during their derivation and/or propagation, which greatly restrict their future therapeutic potential. Although the human-feeder systems, the autogeneic-feeder systems and multiple feederfree systems may be safe for cultivation of undifferentiated hESCs, the derivation procedure free of xeno-products needs to be developed. Here we adopted a non-contact laser-assisted hatching system in combination with sequential culture process to obtain hatched blastocysts as materials for hESC derivation, and derived a well-characterized hESC line, ZJUhES-1, from a laser-assistedly hatched blastocyst of Chinese population without exposure to any nonhuman materials during derivation. The cell line satisfies the criteria of pluripotent hESCs: typically morphological characteristics; the expression of alkaline phosphatase, human telomerase reverse transcriptase and a series of hESC-specific markers including SSEA-3, SSEA-4, TRA-1-60, TRA-1-81, OCT-4, NANOG, REX-1, SOX-2, UTF-1, CONNEXINS 43 and 45, TERF-1 and TERF-2, GLUT-1, BCRP-1/ ABCG-2, GDF3, LIN28, FGF4, Thy1, Cripto1/ TDGF1, AC133 as well as SMAD2/3 and SMAD1/5 which are the signal transducers of TGFa superfamily signaling pathway that is required for maintaining hESC identity; extended proliferative capacity; maintenance of a stable male karyotype after long-term cultivation; robust multiple-lineage developmental potentials to form derivatives of all three embryonic germ layers both in vivo and in vitro. Moreover, the cell line has distinct identity revealed from DNA fingerprinting. In addition, accumulating evidences suggest that directed differentiation of hESCs toward a homogenous population of a specific cell type is prerequisite for their unlimited application in regenerative medicine. However, most published differentiation protocols involving embryoid body formation and the use of coculture stromal cells are poorly defined and inefficient. Starting from monolayer culture, we use all-trans retinoic acid plus FGFs and different culture media to allow for the selective induction of hESCs toward neural, glia and early smooth muscle lineages, demonstrating the pluripotency of ZJUhES-1 in an easy manipulation way.

Keywords: human embryonic stem cells, derivation, hatched blastocysts, laser-assisted hatching system, non-contact laser, directed differentiation

Cell Research (2008) 18:s134. doi: 10.1038/cr.2008.224; published online 4 August 2008

Correspondence: Ming Zhang

E-mail: zhangming_1s@zju.edu.cn 